\title{
BMJ Open Nocturnal emergency department visits, duration of symptoms and risk of hospitalisation among adults with asthma exacerbations: a multicentre observational study
}

\author{
Hideto Yasuda, ${ }^{1,2}$ Yusuke Hagiwara, ${ }^{3}$ Hiroko Watase, ${ }^{4}$ Kohei Hasegawa, ${ }^{5}$ \\ on behalf of the Japanese Emergency Medicine Network Investigators
}

To cite: Yasuda $\mathrm{H}$, Hagiwara $\mathrm{Y}$, Watase $\mathrm{H}$, et al. Nocturnal emergency department visits, duration of symptoms and risk of hospitalisation among adults with asthma exacerbations: a multicentre observational study. BMJ Open 2016;6: e010670. doi:10.1136/ bmjopen-2015-010670

- Prepublication history for this paper is available online. To view these files please visit the journal online (http://dx.doi.org/10.1136/ bmjopen-2015-010670).

Received 4 December 2015 Revised 9 July 2016 Accepted 11 July 2016

CrossMark

For numbered affiliations see end of article.

\section{Correspondence to} Dr Hideto Yasuda; yasuda.hideto@kameda.jp, yasudahideto@me.com

\section{ABSTRACT}

Objectives: We sought to compare the characteristics of patients with asthma presenting to the emergency department (ED) during the night-time with those of patients presenting at other times of the day, and to determine whether the time of ED presentation is associated with the risk of hospitalisation.

Design and setting: A multicentre chart review study of 23 EDs across Japan.

Participants: Patients aged 18-54 years with a history of physician-diagnosed asthma, presented to the ED between January 2009 and

December 2011

Outcome measures: The outcome of interest was hospitalisation, including admissions to an observation unit, inpatient unit and intensive care unit.

Results: Among the 1354 patients $(30.1 \%$ in the night-time group vs $69.9 \%$ in the other time group) included in this study, the median age was 34 years and $\sim 40 \%$ were male. Overall 145 patients $(10.7 \%)$ were hospitalised. Patients in the night-time group were more likely to have a shorter duration of symptoms ( $\leq 3$ hours) before ED presentation than those in the other time group $(25.9 \%$ in night-time vs $13.4 \%$ in other times; $p<0.001)$. In contrast, there were no significant differences in respiratory rate, initial peak expiratory flow or ED asthma treatment between the two groups $(p>0.05)$. Similarly, the risk of hospitalisation did not differ between the two groups $(11.3 \%$ in nighttime vs $10.5 \%$ in other times; $p=0.65$ ). In a multivariable model adjusting for potential confounders, the risk of hospitalisation in the night-time group was not statistically different from the other time group (OR, 1.10; $95 \% \mathrm{Cl} 0.74$ to $1.61 ; \mathrm{p}=0.63$ ).

Conclusions: This multicentre study in Japan demonstrated no significant difference in the risk of hospitalisations according to the time of ED presentation.

\section{INTRODUCTION}

Asthma is an inflammatory disease of the airways and frequently characterised by

\section{Strengths and limitations of this study}

- This is an analysis of a large multicentre chart review study from 23 emergency departments (EDs) across Japan.

- As with observational study, the lack of association between nocturnal ED visits and risk of hospitalisation might be confounded by unmeasured factors, such as the time of onset of asthma exacerbation and institutional variation in resource usage.

- Our inferences may not be generalisable to rural or non-academic EDs or other healthcare settings outside of Japan.

marked changes in symptoms according to a circadian rhythm. More than two-thirds of patients with asthma experience nocturnal exacerbations. ${ }^{1-3}$ It has been speculated that the treatment of patients presenting to the emergency department (ED) with asthma exacerbation may be less successful at the night-time than during the other times of the day because acute asthma severity increases at night. ${ }^{4}$ However, other studies from North America have failed to demonstrate a consistent relationship between nocturnal visits to the ED and poor outcomes, such as an increased risk of hospitalisation. ${ }^{5-8}$ In addition, to the best of our knowledge, the relationship between the time of the ED presentation and acute outcomes of asthma exacerbation has not been studied in other settings (eg, Asian countries).

To address the knowledge gap in the literature, using the data from a multicentre study, we aimed to compare the characteristics of patients presenting to the ED for asthma exacerbation at the night-time (between midnight and 07:59) with those of patients presenting at the other times of the day (between 08:00 and 23:59), and to investigate 
whether the time of ED presentation is associated with the risk of hospitalisation for asthma exacerbation.

\section{METHODS}

\section{Study design and settings}

This is a secondary analysis of a multicentre chart review study to characterise adult patients with asthma exacerbation and their management in 23 EDs across Japan. ${ }^{9}{ }^{10}$ This study was coordinated by the Japanese Emergency Medicine Network (JEMNet) in collaboration with the US-based Emergency Medicine Network (http://www.emnet-usa.org). The JEMNet (http:// jemnet.asia/wp) is a consortium of 23 academic and community hospitals from different geographic regions across Japan. During the period of this study, all of the EDs were staffed by ED-based attending physicians. The study was approved by the Institutional Review Board of each participating centre (see the Acknowledgements section)' and Massachusetts General Hospital.

\section{Study population}

Using the International Classification of Diseases, Tenth Revision, Clinical Modification (ICD-10-CM) code J45.xx, each participating hospital identified all ED visits with a principal ED or hospital discharge diagnosis of asthma from hospital administrative records from 1 January 2009 through 1 December 2011. Patients aged 1854 years, with a history of physician-diagnosed asthma before the index ED visit were eligible for the analysis. We excluded $\left({ }^{1}\right)$ visits made by patients with a history of physician-diagnosed chronic obstructive pulmonary disease (COPD), emphysema or chronic bronchitis, (2) transfer visits, (3) repeat visits by the same patient or (4) visits not largely prompted by an asthma exacerbation. In the case of repeat visits, only the first ED visit during the study period was included in the analysis.

\section{Data collection and measurements}

Onsite chart abstractors reviewed 60 randomly selected ED charts from January to December 2011. If sites had $<60$ charts during this period, the abstractors reviewed eligible charts dating back to January 2009 in order to reach the target. All chart abstractors were emergency physicians or emergency medicine residents who had been trained in a 1-hour online lecture. They were required to complete practice charts, which were assessed against a 'criterion standard'; those whose accuracy was $<80 \%$ per chart had to be retrained.

The following data were abstracted using a standardised form: patients' demographics, chronic asthma characteristics, asthma exacerbation presentation, management in the ED and at discharge, and ED disposition. This standardised form has been used in multiple US-based studies conducted by the Emergency Medicine Network. ${ }^{6-16}$

\section{Outcome measures}

The outcome of interest was hospitalisation, defined by an admission to an observation unit, inpatient unit and intensive care unit.

\section{Statistical analysis}

For the purpose of this analysis, patients were categorised into two groups according to their time at ED presentation: ${ }^{6}$ the night-time (between midnight and 07:59) and the other times (between 08:00 and 23:59). First, the unadjusted association of the time of ED presentation with the patient characteristics and ED management was examined using the $\chi^{2}$ test and Mann-Whitney $\mathrm{U}$ test, as appropriate. Next, to examine the association of the time of ED presentation with the risk of hospitalisation, we fitted a multivariable logistic regression model adjusting for eight potential confounders that were selected based on a priori knowledge and clinical plausibility: age, sex, comorbidity, history of intubation for asthma, history of ED visits over the last year, history of hospitalisations for asthma in the last year and the current use of inhaled corticosteroids and leukotriene modifiers.

Missing indicator methods were used to address missing data. ORs were presented with 95\% CIs. Statistical analyses were performed using JMP V.10.0 software (SAS Institute, Cary, North Carolina, USA) and all tests were two-tailed using a statistical significance value of $\mathrm{p}<0.05$.

\section{RESULTS}

We identified 1380 patients who presented to one of the 23 EDs with asthma exacerbation. Of these, we excluded 26 patients who had missing data on the time of ED presentation (non-analytic cohort), and the remaining 1354 patients $(98 \%)$ had data on the time of ED presentation (analytic cohort), and were included in the current analysis. The analytic and non-analytic cohorts had similar demographics, chronic asthma characteristics, ED presentation and ED disposition (all $\mathrm{p}>0.05$; data not shown).

\section{Demographic characteristics and chronic asthma factors}

Overall, the median age was 35 years (IQR, 26-43 years), $42 \%$ were male and $11 \%$ were hospitalised to the ED. Of 1354 patients in the analytic cohort, $408(30 \%)$ presented to the $\mathrm{ED}$ at the night-time (the night-time group) and $946(70 \%)$ at the other times (the other time group). There were no significant differences in the demographic characteristics, chronic asthma characteristics or current asthma medications between the two groups (table 1).

\section{ED course of patients with asthma exacerbation}

Asthma exacerbation presentation and ED course, according to the time of ED presentation, are shown in table 1. Patients in the night-time group were more 
Table 1 Demographic characteristics, chronic asthma factors and emergency department (ED) course of patients with asthma exacerbation, according to the time of ED presentation

\begin{tabular}{|c|c|c|c|}
\hline Variables & $\begin{array}{l}\text { Midnight-07:59 } \\
\text { (night-time) } \\
\mathrm{n}=408(30 \%)\end{array}$ & $\begin{array}{l}\text { 08:00-23:59 } \\
\text { (other times) } \\
n=946(70 \%)\end{array}$ & p Value \\
\hline \multicolumn{4}{|l|}{ Demographics } \\
\hline Age (year), median (IQR) & $34(26-42)$ & $35(26-43)$ & 0.55 \\
\hline Female sex & $224(55 \%)$ & $571(60 \%)$ & 0.06 \\
\hline Not pregnant* & $165(90 \%)$ & $423(93 \%)$ & 0.32 \\
\hline Comorbidity & $37(9 \%)$ & $114(12 \%)$ & 0.11 \\
\hline Age at the diagnosis of asthma (year), median (IQR) & $19(6-30)$ & $20(9-30)$ & 0.29 \\
\hline Current smoking & $130(45 \%)$ & $268(40 \%)$ & 0.07 \\
\hline \multicolumn{4}{|l|}{ Chronic asthma factors } \\
\hline History of intubation for asthma & $10(4 \%)$ & $11(2 \%)$ & 0.08 \\
\hline History of hospitalisation for asthma & $95(37 \%)$ & $189(33 \%)$ & 0.30 \\
\hline Ever use of systemic corticosteroids & $113(52 \%)$ & $266(50 \%)$ & 0.62 \\
\hline ED visit for asthma in last year & $70(25 \%)$ & $144(21 \%)$ & 0.15 \\
\hline Hospitalised for asthma in last year & $26(8 \%)$ & $52(7 \%)$ & 0.58 \\
\hline Current use of inhaled corticosteroids & $113(29 \%)$ & $290(33 \%)$ & 0.15 \\
\hline Current use of oral methylxanthines & $49(12 \%)$ & $99(11 \%)$ & 0.54 \\
\hline Current use of leukotriene modifiers & $51(13 \%)$ & $96(11 \%)$ & 0.30 \\
\hline \multicolumn{4}{|l|}{ Presentation and ED course } \\
\hline Duration of symptoms before ED arrival & & & $<0.001$ \\
\hline$\leq 3$ hours & $103(26 \%)$ & $122(13 \%)$ & \\
\hline 4-11 hours & $103(26 \%)$ & $181(20 \%)$ & \\
\hline $12-24$ hours & 77 (20\%) & $219(24 \%)$ & \\
\hline $1-3$ days & $71(18 \%)$ & $244(27 \%)$ & \\
\hline 4-7 days & $24(6 \%)$ & $93(10 \%)$ & \\
\hline >7 days & $19(5 \%)$ & $51(6 \%)$ & \\
\hline Prehospital treatment & $45(12 \%)$ & $110(12 \%)$ & 0.64 \\
\hline Initial respiratory rate (breaths/min), median (IQR) & $21(18-25)$ & $20(18-24)$ & 0.13 \\
\hline Initial oxygen saturation on room air (\%), median (IQR) & $96(93-98)$ & $96(94-98)$ & 0.03 \\
\hline Initial PEF (L/min), median (IQR)† & $205(143-258)$ & $200(150-290)$ & 0.61 \\
\hline $\begin{array}{l}\text { Number of inhaled } \beta \text {-agonists in } 1 \text { hour from ED } \\
\text { presentation, median (IQR) }\end{array}$ & $1(1-2)$ & $1(1-2)$ & 0.09 \\
\hline Number of inhaled $\beta$-agonists in ED, median (IQR) & $1(1-2)$ & $1(1-2)$ & 0.38 \\
\hline Systemic corticosteroids in ED & $160(39 \%)$ & $409(43 \%)$ & 0.17 \\
\hline Intravenous magnesium in ED & $5(1 \%)$ & $9(1 \%)$ & 0.65 \\
\hline ED disposition & & & 0.62 \\
\hline Observation unit & $5(1 \%)$ & $9(1 \%)$ & \\
\hline Hospital ward & $37(9 \%)$ & $81(9 \%)$ & \\
\hline Intensive care unit & $4(1 \%)$ & $9(1 \%)$ & \\
\hline Sent homeł & $356(87 \%)$ & $841(89 \%)$ & \\
\hline Others (eg, left against medical advice) & $6(2 \%)$ & $6(1 \%)$ & \\
\hline \multicolumn{4}{|c|}{$\begin{array}{l}\text { Data were presented as number (\%) unless otherwise indicated. } \\
{ }^{*} \text { Analysed for } 183(44.9 \%) \text { female patients in the night-time group and } 457(48.3 \%) \text { female patients in the other group. } \\
\text { †Analysed for } 32(7.8 \%) \text { patients in the night-time group and } 77(8.1 \%) \text { patients in the other time group with initial PEF available. } \\
\text { fAnalysed for } 356(87.3 \%) \text { patients in the night-time group and } 841(88.9 \%) \text { patients in the other time group who were discharged to home. } \\
\text { ED, emergency department; PEF, peak expiratory flow. }\end{array}$} \\
\hline
\end{tabular}

likely to have a shorter duration of symptoms ( $\leq 3$ hours) prior to their ED presentation compared to those in the other time group (26\% vs $13 \%$; $\mathrm{p}<0.001)$. However, there were no significant differences in the presentation (eg, respiratory rate, initial peak expiratory flow) or acute asthma treatment in the ED between the groups. Similarly, the proportion of the patients hospitalised from the ED did not differ significantly between the two groups $(11 \%$ vs $11 \%$; $\mathrm{p}=0.65)$. Likewise, in the multivariable logistic regression model adjusting for eight potential confounders, the time of ED presentation was not associated with the risk of hospitalisation (OR for night-time vs other times, $1.10 ; 95 \%$ CI 0.74 to 1.61 ; $\mathrm{p}=0.63$; table 2 ).

\section{DISCUSSION}

In this multicentre study of $1354 \mathrm{ED}$ patients with asthma exacerbation, $\sim 30 \%$ of the patients presented to the ED at the night-time. These patients presented to 
Table 2 Unadjusted and adjusted association of time of emergency department presentation with risk of hospitalisation

\begin{tabular}{lllll}
\hline Time of ED presentation & $\begin{array}{l}\text { Unadjusted analysis } \\
\text { OR }(\mathbf{9 5 \%} \text { Cl) }\end{array}$ & p Value & $\begin{array}{l}\text { Multivariable analysis* } \\
\text { OR }(\mathbf{9 5 \%} \text { Cl) }\end{array}$ & p Value \\
\hline 08:00-23:59 & 1.00 (reference) & - & 1.00 (reference) & - \\
Midnight-07:59 & $1.09(0.75$ to 1.57$)$ & 0.65 & $1.10(0.74$ to 1.61$)$ & 0.63 \\
\hline
\end{tabular}

*Multivariable analysis adjusted for age, sex, comorbidity, history of intubation for asthma, history of admissions for asthma in the last year, history of ED visits over the last year, current use of inhaled corticosteroids and leukotriene modifiers.

$E D$, emergency department.

the ED with a shorter duration of symptoms compared to those who presented to the ED at the other times. In contrast, our data did not indicate any circadian difference in the risk of hospitalisation in ED patients with asthma exacerbations.

\section{Results in context}

Despite the clinical importance, few studies have examined the relationship of clinical characteristics of asthma exacerbation with nocturnal asthma in the recent years. Several studies have reported that patients with nocturnal asthma had a higher morbidity and inhospital mortality compared to others. ${ }^{4}{ }^{17-19}$ In contrast, in agreement with our observations, previous North American studies of ED patients with asthma exacerbation did not find a significant association between the time of ED presentation and the risk of hospitalisation. ${ }^{6-8}$ For example, Brenner et $a l^{6}$ by analysing the data of a multicentre prospective cohort of $2494 \mathrm{ED}$ adult patients (aged 18-54 years) with asthma exacerbation in the USA and Canada, reported no association between nocturnal visits and risks of hospitalisation. The reasons for the apparent discrepancies between the studies are likely multifactorial-for example, the differences in study design, patient population, setting or any combination of these factors. Indeed, the aforementioned studies that reported a higher morbidity in patients with nocturnal asthma were conducted in the inpatient setting ${ }^{17} 19$ while the studies that demonstrated the lack of morbidity association, ${ }^{6-8}$ including our work, were conducted in the ED setting. These ED data from different healthcare systems (the USA, Canada and Japan) collectively suggest that nocturnal ED visits for asthma exacerbation may not be associated with the more-severe acute asthma outcomes in ED patients, unlike such cases in hospitalised patients.

Alternatively, non-biological factors, such as access to healthcare including primary and asthma specialist care, patient health behaviours, use of long-term controllers and chronic and acute severity of asthma, also are likely to have contributed to the discrepancy. Indeed, in our study, the proportion of inhaled corticosteroid use prior to the ED visit (32\%) was higher than the previous studies, although it remained suboptimal. The higher proportion of patients with long-term controller use in our study might also have affected the acute asthma severity and response to therapy, and further contributed to the absence of significant association between the nocturnal ED visits for asthma exacerbation and the risk of hospitalisation. Our multicentre study of a large number of ED adults builds on these prior studies that examined the risk of hospitalisation by time of ED presentation and extends them by conducting a multicentre study in Japan.

\section{Potential limitations}

We acknowledge several potential limitations of this study. First, this multicentre study relied on chart review for data collection, and hence errors in data measurement are possible. However, we used a previously applied standardised data collection system with uniform definitions and rigorous training, which achieved a high interobserver agreement ( $\kappa$-statistics, 0.56-1.00 (moderate-to-perfect agreement) ). ${ }^{9}$ Second, our study had no information on the reasons for nocturnal ED visits. Third, as with any observational study, the lack of association between nocturnal ED visits and risk of hospitalisation might be confounded by unmeasured factors, such as the time of onset of asthma exacerbation and institutional variation in resource usage. Indeed, in this multicentre study, we did not standardise criteria for hospitalisation across the 23 participating EDs. Fourth, the limited age range (18-54 years) in this study may limit the generalisability of our findings. However, we chose this age range to limit a potential contamination of older patients with COPD or asthma-COPD overlap syndrome that may bias the inferences. Finally, our sample consisted predominantly of urban, academic EDs in Japan. Our study population had a relatively high chronic severity burden (eg, 22\% of patients had at least one ED visit for asthma in the preceding year) and high acute severity (eg, 8\% resulted in hospitalisation), although these proportions were lower than those in the recent multicentre study from the USA. ${ }^{20}$ In addition, the participating EDs provided acute asthma care that was imperfectly concordant to the recommendations in the asthma guidelines. ${ }^{9}$ Therefore, our inferences may not be generalisable to rural or non-academic EDs or other healthcare settings outside of Japan. Nevertheless, multiple studies arrived at a similar conclusion (ie, no association between the time of ED presentation and risk of hospitalisation), despite differing populations and healthcare settings, ${ }^{6-8}$ potentially enhancing the generalisability of our inferences. 


\section{CONCLUSION}

In this multicentre analysis of $1354 \mathrm{ED}$ patients with asthma exacerbation, we found that many patients presented to the ED at the night-time. Our data also demonstrated no significant difference in the risk of hospitalisations according to the time of ED presentation. The reasons for nocturnal ED visits for asthma exacerbation are multifactorial-for example, acute asthma severity, access to the healthcare system and patient behaviour. Our data should facilitate further investigation to disentangle the complex web of these factors.

\section{Author affiliations}

${ }^{1}$ Intensive Care Unit, Department of Emergency and Critical Care Medicine, Japanese Red Cross Musashino Hospital, Tokyo, Japan

${ }^{2}$ Department of Intensive Care Medicine, Kaneda Medical Center, Chiba, Japan

${ }^{3}$ Department of Pediatric Emergency and Critical Care Medicine, Tokyo

Metropolitan Children's Medical Center, Tokyo, Japan

${ }^{4}$ Department of Radiology, University of Washington, Seattle, Washington, USA

${ }^{5}$ Department of Emergency Medicine, Massachusetts General Hospital, Harvard Medical School, Boston, Massachusetts, USA

Acknowledgements The authors thank the participating investigators for their ongoing dedication to emergency medicine research.

Collaborators The site principal investigators for the Japanese Emergency Medicine Network were Yukikazu Awaya, MD (Yahata Municipal Hospital, Kitakyushu, Fukuoka), Tadahiro Goto, MD (University of Fukui Hospital, Eiheiji, Fukui), Nao Hanaki, MD ( Japanese Redcross Nagoya Second Hospital, Nagoya Aichi), Takuro Hayashi, MD (Kobe City Medical Center General Hospital, Kobe, Hyogo), Taichi Imamura, MD (Shonan Kamakura General Hospital), Yusuke Kodama, MD (Kyoritsu General Hospital, Nagoya, Aichi), Takashiro Kondo, MD (Nagoya Ekisaikai Hospital, Nagoya, Aichi), Yasuaki Koyama, MD (St. Marianna University Hospital, Kawasaki, Kanagawa), Tomomichi Matsuda, MD (Sapporo East Tokushukai Hospital, Sappro, Hokkaido), Nobuhiro Miyamae, MD (Rakuwakai Otowa Hospital, Kyoto, Kyoto), Miki Morikawa, MD (Juntendo University Urayasu Hospital, Urayasu, Chiba), Hideya Nagai, MD (Fukui Prefectural Hospital, Fukui, Fukui), Masahumi Ohta, MD (Kensei Hospital, Tsugaru, Aomori), Hiroshi Okamoto, MD (Kurashiki Central Hospital, Kurashiki, Okayama), Yuichi Okano, MD ( Japanese Redcross Kumamoto Hospital, Kumamoto, Kumamoto), Masashi Okubo, MD (Okinawa Prefectural Chubu Hospital, Uruma, Okinawa), Yutaka Saito, MD (Kawasaki City Hospital Kawasaki, Kawasaki), Hiroyuki Suzuki, MD (Fukuoka Tokushukai Hospital, Kasuga, Fukuoka), Sei Takahashi, MD (Kinikyo Chuo Hospital, Sappro, Hokkaido), Kotaro Takebe, MD (Kyoto Prefectural University Hospital, Kyoto, Kyoto), Kenzo Tanaka, MD, MPH (Kameda Medical Center, Kamogawa, Chiba), and Yuki Sato, MD (Nigata City General Hospital, Nigata, Nigata).

Contributors HY contributed in the study concept and design, analysis and interpretation of the data and drafting of the manuscript. YH and HW contributed in the study concept and design, acquisition of the data and critical revision of the manuscript for important intellectual content. KH contributed in the study concept and design, interpretation of the data and critical revision of the manuscript for important intellectual content.

Funding This study was supported by a grant from St. Luke's Life Science Institute (Tokyo, Japan).

Competing interests $\mathrm{KH}$ has received research support from Teva.

Patient consent Obtained.

Provenance and peer review Not commissioned; externally peer reviewed.

Data sharing statement No additional data are available.
Open Access This is an Open Access article distributed in accordance with the Creative Commons Attribution Non Commercial (CC BY-NC 4.0) license, which permits others to distribute, remix, adapt, build upon this work noncommercially, and license their derivative works on different terms, provided the original work is properly cited and the use is non-commercial. See: http:// creativecommons.org/licenses/by-nc/4.0/

\section{REFERENCES}

1. Strunk RC, Sternberg AL, Bacharier LB, et al. Nocturnal awakening caused by asthma in children with mild-to-moderate asthma in the childhood asthma management program. J Allergy Clin Immunol 2002;110:395-403.

2. Turner-Warwick M. Epidemiology of nocturnal asthma. Am J Med 1988;85:6-8.

3. Raherison C, Abouelfath A, Le Gros V, et al. Underdiagnosis of nocturnal symptoms in asthma in general practice. J Asthma 2006;43:199-202.

4. Braido F, Baiardini I, Ghiglione V, et al. Sleep disturbances and asthma control: a real life study. Asian Pac J Allergy Immunol 2009;27:27-33.

5. Buff DD, Bavli SZ, Bloch $\mathrm{MH}$, et al. Circadian patterns of emergency asthma presentations: implications for staffing and treatment. Respir Care 1995;40:624-30.

6. Brenner BE, Chavda KK, Karakurum MB, et al. Circadian differences among 4,096 emergency department patients with acute asthma. Crit Care Med 2001;29:1124-9.

7. Baibergenova A, Thabane L, Akhtar-Danesh N, et al. Patient characteristics associated with nocturnal emergency department visits for asthma. J Asthma 2006:43:469-75.

8. Karras DJ, D'Alonzo GE, Heilpern KL. Is circadian variation in asthma severity relevant in the emergency department? Ann Emerg Med 1995;26:558-62.

9. Hasegawa K, Chiba T, Hagiwara Y, et al. Quality of care for acute asthma in emergency departments in Japan: a multicenter observational study. J Allergy Clin Immunol Pract 2013;1:509-15. e1-3.

10. Watase $\mathrm{H}$, Hagiwara $\mathrm{Y}$, Chiba $\mathrm{T}$, et al. Multicentre observational study of adults with asthma exacerbations: who are the frequent users of the emergency department in Japan? BMJ Open 2015;5: e007435.

11. Patel SN, Tsai CL, Boudreaux ED, et al. Multicenter study of cigarette smoking among patients presenting to the emergency department with acute asthma. Ann Allergy Asthma Immunol 2009;103:121-7.

12. Ramnath VR, Clark S, Camargo CA Jr. Multicenter study of clinical features of sudden-onset versus slower-onset asthma exacerbations requiring hospitalization. Respir Care 2007;52:1013-20.

13. Banerii A, Clark S, Afilalo $M$, et al. Prospective multicenter study of acute asthma in younger versus older adults presenting to the emergency department. J Am Geriatr Soc 2006;54:48-55.

14. Pollack CV, Jr., Pollack ES, Baren JM, et al. A prospective multicenter study of patient factors associated with hospital admission from the emergency department among children with acute asthma. Arch Pediatr Adolesc Med 2002;156:934-40.

15. Weber EJ, Silverman RA, Callaham ML, et al. A prospective multicenter study of factors associated with hospital admission among adults with acute asthma. Am J Med 2002;113:371-8.

16. Emerman CL, Cydulka RK, Crain EF, et al. Prospective multicenter study of relapse after treatment for acute asthma among children presenting to the emergency department. $J$ Pediatr 2001;138:318-24.

17. Cochrane GM, Clark JH. A survey of asthma mortality in patients between ages 35 and 64 in the Greater London hospitals in 1971. Thorax 1975;30:300-5.

18. Robertson CF, Rubinfeld AR, Bowes G. Deaths from asthma in Victoria: a 12-month survey. Med J Aust 1990;152:511-17.

19. Hetzel MR, Clark TJ, Branthwaite MA. Asthma: analysis of sudden deaths and ventilatory arrests in hospital. Br Med $\mathrm{J}$ 1977;1:808-11.

20. Hasegawa K, Sullivan AF, Tovar Hirashima E, et al. A multicenter observational study of US adults with acute asthma: who are the frequent users of the emergency department? J Allergy Clin Immunol Pract 2014;2:733-40. 